See discussions, stats, and author profiles for this publication at: https://www.researchgate.net/publication/235922663

\title{
Microfluidic tools to investigate pathologies in the blood microcirculation
}

Article in International Journal of Nanotechnology · February 2012

DOI: 10.1504/IJNT.2012.045340

CITATIONS

10 authors, including:

$$
\text { Pascal Preira }
$$

IPBS - Institut de Pharmacologie et de Biologie Structurale

7 PUBLICATIONS 158 CITATIONS

SEE PROFILE

(a)

Philippe Robert

Assistance Publique Hôpitaux de Marseille

29 PUBLICATIONS 306 CITATIONS

SEE PROFILE
150

Thomas Leoni

Aix-Marseille Université

24 PUBLICATIONS 316 CITATIONS

SEE PROFILE

Forel jean marie

Assistance Publique Hôpitaux de Marseille

114 PUBLICATIONS 3,204 CITATIONS

SEE PROFILE

Some of the authors of this publication are also working on these related projects:

Atomic point contacts View project 


\title{
Microfluidic tools to investigate pathologies in the blood microcirculation
}

\author{
Pascal Preira, Thomas Leoni, \\ Marie-Pierre Valignat, \\ Annemarie Lellouch \\ and Philippe Robert \\ Université de la Méditerranée, \\ Adhesion \& Inflammation, \\ INSERM U600-CNRS UMR6212, Case 937, \\ 163 Avenue de Luminy, \\ F-13009 Marseille, France \\ Fax: +33 491828851 \\ E-mail: pascal.preira@inserm.fr \\ E-mail: thomas.leoni@inserm.fr \\ E-mail: marie-pierre.valignat@inserm.fr \\ E-mail: annemarie.lellouch@inserm.fr \\ E-mail: philippe.robert@inserm.fr
}

\section{Jean-Marie Forel and Laurent Papazian}

Unité de Recherche sur les Maladies Infectieuses

et Tropicales Emergentes (URMITE) UMR6236,

CNRS/Université Aix-Marseille 2,

Hôpital Nord, Chemin des Bourrely,

13015 Marseille Cedex 20, France

E-mail: jeanmarie.forel@ap-hm.fr

E-mail: laurent.papazian@ap-hm.fr

\section{Guillaume Dumenil}

\section{Université Paris Descartes,}

Faculté de Médecine Paris Descartes,

Paris F-75006, France

and

PARCC, Centre de Recherche Cardiovasculaire à l'HEGP, Inserm U970, 56 rue Leblanc,

75015 Paris, France

E-mail: guillaume.dumenil@inserm.fr 


\title{
Pierre Bongrand and Olivier Théodoly*
}

Université de la Méditerranée, Adhesion \& Inflammation, INSERM U600-CNRS UMR6212, Case 937, 163 Avenue de Luminy, F-13009 Marseille, France

Fax: +33 491828851

E-mail: pierre.bongrand@inserm.fr

E-mail: olivier.theodoly@inserm.fr

${ }^{*}$ Corresponding author

\begin{abstract}
We show how microfluidics technology can be used to fabricate simple and innovative biomimetic tools to shed new light on physiopathological events occurring in the blood microcirculation. Examples of applications are given in the context of the acute respiratory distress Syndrome (ARDS), an inflammatory disease of the lung triggered by a massive arrest of white blood cells in the lung microvasculature. The main challenge consists in building relevant micro-devices to reproduce key biological characteristics of blood capillaries. We present a series of tools that permit us to decouple the role of the multiple parameters involved in complex biological events. Straight narrow channels with non-adherent walls are used to characterise the passage of a cell in $4 \mu \mathrm{m}$ wide constrictions in the absence of adhesion, whereas channels covered by endothelial cells allow a quantitative measurement of cell adhesion in the absence of mechanical constraints. We show that incubation of white blood cells in sera of ARDS patients increases their stiffness, confirming the role of stiffness on the abnormal sequestration of white blood cells, whereas we could not bring to light a significant adhesion increase. The multiple branches and constrictions of the blood microvasculature network are mimicked here by series of interconnected crenelled constrictions with different symmetries. In symmetric crenels, cells adopt a stable deformed shape after a few constrictions and travel fast through successive constrictions with a constant orientation. In asymmetric channels, cell orientation and trajectory are perturbed between two constrictions. Unfavourable orientations upon entry can yield temporary or even definitive arrests with the stiffest cells. Finally, we present a new artificial micro-vessel with porous walls to mimic the porosity of real blood vessels. This new tool is useful to observe directly under a microscope the late stages of inflammation in the microvasculature such as immune cells transmigration, or the infection of a micro-vessel by pathogenic bacteria.
\end{abstract}

Keywords: microfluidics; microvasculature; cell mechanics; cell adhesion; cell migration; cell transmigration; respiratory distress syndrome; meningitis.

Reference to this paper should be made as follows: Preira, P., Leoni, T., Valignat, M-P., Lellouch, A., Robert, P., Forel, J-M., Papazian, L., Dumenil, G., Bongrand, P. and Théodoly, O. (2012) 'Microfluidic tools to investigate pathologies in the blood microcirculation', Int. J. Nanotechnol., Vol. 9, Nos. 3-7, pp.529-547.

Biographical notes: Pascal Preira is a Graduate Student at the Aix-Marseille University, performing microfluidics experimentation to probe the rheology of circulating blood cells. 
Thomas Leoni obtained his $\mathrm{PhD}$ in Condensed Matter Physics from the Aix-Marseille University in 2009. As a post-doc in 2010 in the Laboratory Adhesion and Inflammation in Marseilles, France, he has developed biomimetic devices for blood capillaries with microchannels covered with endothelial cells. He is now a Research Associate in CEMES, Toulouse, France, where he works on charge transfer on single molecule.

Marie-Pierre Valignat is an Assistant Professor in Biophysics at the Aix-Marseille University, France. She received a $\mathrm{PhD}$ in Physics from the University of Paris-6 in 1994. Her main fields of interest concern the wetting of complex fluids, the development of optical techniques to observe nanoscopic thin films and the spreading and migration of living cells.

Annemarie Lellouch is a CNRS Staff Scientist at the Laboratory Adhesion and Inflammation in Marseilles, France. She received her $\mathrm{PhD}$ in Biological Chemistry from the Massachusetts Institute of Technology in 1992. Her current research interests include the study of biochemical and mechanical signalling phenomena involved in $\mathrm{T}$ lymphocyte motility with an emphasis on the design and application of fluorescent biosensors for live cell imaging of biochemical events in motile cells.

Philippe Robert is an Assistant Professor in Immunology at Aix-Marseille University, France. He received an MD in Clinical Immmunology in 2007, and a $\mathrm{PhD}$ in Immunology in 2009. His main field is the study of immunological ligand-receptor interaction with laminar flow chamber, both at the molecular and at the cellular levels.

Jean-Marie Forel is an Associate Professor of Intensive Care at Marseille Medical School, Aix-Marseille Université, Marseilles, France.

Laurent Papazian is a Professor of Intensive Care at Marseille Medical School, Head of the Intensive Care Unit of North Hospital, Marseille, and Head of a team from the URMITE CNRS-UMR 6236 research unit, Aix-Marseille Université, Marseille, France. His main research interests are the physiopathology, diagnosis and treatment of ventilator-associated pneumonia and the physiopathology and treatment of the acute respiratory distress syndrome.

Guillaume Dumenil is a Microbiologist working at INSERM. He is specialised in the study of host-pathogen interactions taking place during infectious diseases. His main field of interest is currently infections taking place in the context of blood vessels leading to septicaemia and meningitis.

Pierre Bongrand is a Professor of Immunology at Marseille Medical School, Head of the Immunology Laboratory of Conception hospital and Director of the Laboratory Adhesion and Inflammation. He was trained in physics at the Ecole Normale Supérieure (Paris, 1968-1972) and in medicine at Paris and Marseille Universities (1966-1974). His main research interests are the study of biomolecule interactions and immune cell activation at interfaces.

Olivier Théodoly is a Researcher in the French National Research Council (CNRS). He was trained in physics and chemistry at ESPCI-Paristech (France), obtained his $\mathrm{PhD}$ in Physics from the University of Paris 6 in 1999. His professional start was devoted to polymer physics at interfaces in UC-Berkeley in 2000-2001 as a post-doctoral fellow and in the academics/industry joined Laboratory between the CNRS, Princeton University, and RHODIA INC. 
as a CNRS researcher in 2002-2005. Since 2006, he turned his main research interests towards the interface between physics, biology and medicine in the Laboratory Adhesion and Inflammation. His current research concerns the study of the blood microcirculation and the properties of deformation, migration and transmigration of circulating cells.

\section{Introduction}

Medical research has greatly benefited from the recent development of medical imagery tools (magnetic resonance imaging, tomography, nuclear and positrons imagery) that yield fascinating 3D images from the scale of the whole body down to organ details. However, the limited spatial resolution of these techniques precludes observations of phenomena at the cellular level. Optical microscopy remains the most powerful tool to investigate the micrometre-scale living world and many sophisticated techniques (phase contrast [1], fluorescence, DIC [2,3], two photons confocal microscopy [4], Total Internal Reflection Fluorescence Microscopy [5], Ultra-high resolution microscopy [6]) have been developed to probe the cellular activity from the tissue level down to the molecular scale. Unfortunately, the use of optical microscopy on the human body in medicine is strongly limited owing to the diffusion of light by biological matter that restricts penetration of light in the body to a few microns. Non-intrusive microscopy observations on humans are therefore limited to a superficial region that is at most a few hundreds of micrometres deep, whereas fibroscopy imagery allows observation of inner body parts but with poor resolution. The internal microscopic human body is still largely inaccessible, which puts important limitations on medical progress. Biological investigations at the microscopic level can resort to animal experimentation, where surgical windows are employed to access the deep organs [7] but this practice is ethically questionable, expensive and not applicable to those cases where human pathologies have no relevant animal model. A new approach has recently emerged, which consists in fabricating in vitro models to study microscopic internal biological functions of interest directly under a microscope. This approach takes advantage of the advances in microfabrication and microfluidics technologies [8-10]. The coupling of micro-devices with different microscopy observations modes permits one to design new tools with which to explore biological events from the cellular to the nanometre scale.

In this work, we focus on pathological events occurring in the blood microcirculation. Blood vasculature ramifies in the organs into a network of narrow capillaries with diameters as small as $2 \mu \mathrm{m}$, providing a large surface area for efficient exchange of gas and nutrients between blood and organ tissues. Several specific characteristics of the microvasculature make it a special location for pathological events in the blood system. First, capillaries are remarkably small when compared with cells in the blood stream, i.e., blood cells or cancer circulating cells, which have a typical size of approximately $10 \mu \mathrm{m}$. Circulating cells must, therefore, strongly deform to pass through the microvasculature, which puts their mechanical properties to the test. Second, trafficking cells in capillaries are in close contact with the microvascular endothelium during their passage, which favours cell-endothelium interaction and potentially adhesion. Third, the hydrodynamic shear stress in the bloodstream reaches its highest values in arterioles and capillaries [11], 
applying important stresses on endothelial and arrested cells, like immune cells or pathogens. Last, the intimate contact between the microvasculature and surrounding organ tissues favours the sensitivity of immune cells to inflammatory signals and the extravasation of immune cells towards inflammatory zones. This set of characteristics explains why certain pathological events occur specifically in the blood microcirculation. It has been recognised that circulating cells are blocked specifically in the microvasculature owing to an increase in stiffness or adhesion during pneumonia [12], sepsis [13], ARDS [14] and metastasis [15,16]. Conversely, pathologic low affinity of lymphocytes with microvascular endothelium results into immunological deficiency diseases [17,18]. Also, transient flow arrest in the microvasculature can favour opportunistic adhesion of pathogens, like in meningitis infection [19], and abnormal extravasation is known to be responsible for pathological infiltration of organs in certain lymphomas [20]. In this context, the development of new micro-fabricated tools to reproduce in vitro the series of events occurring in vivo in the microvasculature represents a new promise for research on microscopic mechanisms involved in a wide range of diseases [21].

The main challenge in a microfluidic study of the blood microcirculation consists first in building relevant micro-devices that reproduce the key biological characteristics of blood capillaries. An idealistic reconstruction of blood micro-vessels should encompass a lumen with physiological micrometric diameter, surrounded by flexible and porous walls covered with layers of endothelial cells, muscular cells and extracellular matrix tissue. Such a complex model does not yet exist, although models that have been developed progressively approach this ultimate goal. Straight synthetic channels with synthetic non-adherent and non-porous walls of micrometric cross section have been used to investigate the passage of single cells in a constriction [22-27], and their 3D motility in confined environment [28-30]. Several papers have reported experiments with channels covered by endothelial cells [31-34] and mainly used to investigate the characteristics of endothelial cells under hydrodynamic stress [35,36], or the intravascular adhesion of white blood cells [35] and metastatic cancer cells [37]. A recent paper has introduced side micro-channels perpendicular to the main flow chamber that provides porosity to vessel walls [38]. Although this work describes a flow chamber that is too large to mimic the minute dimension of a micro-vessel, the device has proven useful to measure the permeability of the endothelial barriers vs. hydrodynamic stress. Porous synthetic walls have also been created using rectangular channels with two walls made of a hydrogel, and used to investigate cellular morphogenesis [39]. Very recently, a sophisticated biomimetic device has been fabricated that reconstitutes the complex multiple tissue of the lung alveolus [40], comprising both an endothelial and an epithelial layer. This device is also mechanically active, which permits one to apply a mechanical stress on the tissues to reconstitute the constraints applied in vivo by the dilation of alveolus upon respiration.

In this paper, we present a series of microfluidic tools for investigating key pathologic steps occurring in the microvasculature. We focus on potential applications linked to a pulmonary disease called ARDS. ARDS was defined in 1967 as a bilateral pulmonary inflammation following severe trauma that is not necessarily linked to the lungs. ARDS was identified based on the important statistics on trauma yielded by 20th century military casualties [41]. In nowadays civil life, ARDS concerns $7 \%$ of patients in intensive care units and the death rate is around $40 \%$ [42]. In the $1980 \mathrm{~s}$, the convergence 
from traumatisms of multiple origins to a pulmonary disease was attributed to the massive arrest of white blood cells in the lung microvasculature, which was systematically detected on deceased patients [14]. Intensive research has since been undertaken to understand whether pathological arrests of white blood cells in the lungs, also observed in sepsis or pneumonia, were due to an increase in stiffness or adhesion. In sepsis, an increase in both cell stiffness $[43,44]$ and adhesion $[45,46]$ has been reported, whereas in pneumonia a notable increase in stiffness [12] has been identified with no significant role of adhesion [12,45]. Concerning ARDS, it seems quite clear that cell stiffness plays a role $[22,43]$. However, in previous studies, measurements on patients' cells may include an experimental bias because the cells obtained from the population of circulating cells may not be representative of the whole cell population since the relevant cells to examine are trapped in the microvasculature. Finally, the causes of abnormal leucocyte stiffness in ARDS are still obscure, although several reports have demonstrated that inflammatory mediators could increase leucocyte stiffness. Moreover, there are no data on the role played by adhesion in cell arrest. In this paper, we will use our microfluidic tools to shed new light on the role of leucocyte stiffness and adhesion properties in ARDS. To avoid the bias of working with patient cells, we will use a well-established monocytic cell line and incubate these reference cells in sera of patients with ARDS.

A first family of devices concerns microfluidic circuitry with non-adherent walls. These devices put to the test the rheological properties of circulating cells in the absence of adhesion effects. A straight constriction device allows us to measure parameters such as the entry time, the cell friction in the constriction, the unfolding of cell membrane during the capillary transit through a constriction and the shape recovery kinetics after passage in the constriction. Straight channels are not representative of real microvasculature pathways comprising a succession of capillary segments that impose repetitive deformations to the cell during transit. We use here devices with multiple constrictions in series to investigate the passage of stiff cells in intricate micro-circuitries and we test the role of the channels' symmetry on transit time. A second family of devices concerns microfluidic channels with potentially adherent walls covered by endothelial cells. Such flow chamber devices are useful to quantify the adhesivity of cells on vessel endothelium in the absence of mechanical constraints and also to investigate the migration modes of leucocytes on an endothelium. Finally, we present a new family of micro-vessels with porous walls. These devices are aimed at measuring the permeability of an endothelium to both fluids and cells vs. different hydrodynamic conditions or during an infection by a pathogen. These micro-vessels are also adapted to directly observing the detailed mechanism of white blood cells extravasation out of the vessel lumen into surrounding tissues under a microscope.

\section{Materials and methods}

Micro-device fabrication. All microfluidic devices were built using conventional microfabrication techniques developed for microfluidics [47]. Microfluidic channel designs were drawn using Clewin software (WieWeb Software, Hengelo, The Netherlands) to generate a chromium mask (Toppan Photomask, Corbeil Essonnes, France). A positive mould was created with SU-8 2000 negative resins (Microchem, Newton, MA, USA) spin-coated on silicon wafers (Siltronix, Archamps, France) and 
exposed through the chromium mask in 'hard contact' mode with a photolithography device (Suess Microtec, Munich, Germany). Replicas of the positive moulds are obtained made with polydimethylsiloxane (PDMS) (Sylgard 184 Silicone Elastomer Kit, Dow Corning, Midland, USA). Ports to plug inlet and outlet reservoirs were punched with a gauge needle and the devices were finalised by sealing the PDMS piece on a $170 \mathrm{~mm}$ thick glass coverslip via $\mathrm{O}_{2}$-plasma activation (Harricks plasma) of both surfaces.

Fluid manipulation. Fluid reservoirs were connected to the microfluidic device with polyethylene tubings (Scientific Commodities Inc., Lake Havasu City, AZ, USA) and attached to linear translation stages mounted on vertical optical rails (Micro-controle Spectraphysics S.A., Evry, France). Pressure drops across the microchannels were controlled by varying the height of the fluid macro-reservoirs (Figure 1(A)). In the text, we do not report the pressure drop $\Delta P$ across the whole circuitry but the local pressure drop in the portion of the microcircuitry of interest. $\Delta P$ is the pressure drop between the inlet and outlet of the $4 \times 15 \times 2245 \mu \mathrm{m}^{3}$ channel for the single constriction device, and of 50 crenels in series for the multiple constrictions device. The simple method for single-cell experimentation described elsewhere [48] was used to select and manipulate cells without stress, and to exclude all undesired super-micronic particles.

Figure 1 Anti-adherent constriction channels for cell stiffness measurement. (A-B) Image sequences of a leucocyte THP-1 cell during entry into a $4 \times 15 \mu \mathrm{m}^{2}$-wide constriction at a local pressure $\Delta P=100$ Pa before (A) and after (B) incubation in a serum of ARDS patient for $1 \mathrm{~h}$; (i) contact, (ii) development of a projection in the constriction, and (iii) completed entry. The scale bar represents $10 \mu \mathrm{m}$ and $t$ indicates the time after first contact with the entry. (C) Entrance time ETs vs. local pressure drop $\Delta P$ for THP-1 cells before (hollow dots) and after (filled dots) incubation in a serum of ARDS patient for 1 hour. Error bars represent standard error SE $(n=20)$

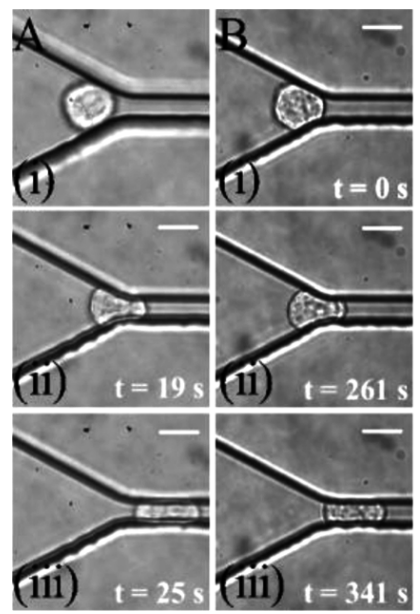

$\mathrm{C}$

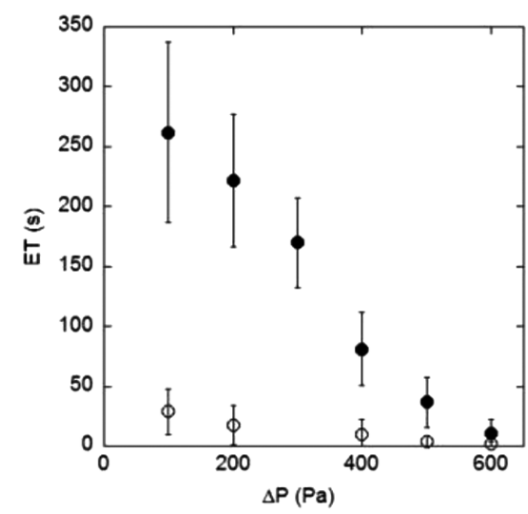

Microscopy set-up. Observations were taken with an inverted microscope (Zeiss AxioObserver 200) equipped for fluorescence and phase contrast microscopy (Plan-Neofluar 100X/1.30 Oil Ph3), and reflection interference contrast microscopy (Antiflex EC Plan-Neofluar 63X/1.25 Oil Ph3). Video sequences are recorded with a camera COHU (model 4912-5000) at 24 frames per second. 
Channel surface treatment. To obtain anti-adherent walls, the channels were incubated with a 5\% Pluronic F108 solution (BASF, Mount Olive NJ, USA) for $2 \mathrm{~h}$. The treatment for leucocyte integrin-specific adhesion in channels was incubation with Fc-ICAM-1 (R\&D Systems) at $10 \mu \mathrm{g} / \mathrm{ml}$ in PBS at $4^{\circ} \mathrm{C}$ overnight, followed by incubation with a blocking solution (BSA 1.25\% and Pluronic F108 1.5\%) for 30 min, and finally rinsed with HBSS supplemented with $5 \mathrm{mM}$ Hepes and $5 \mathrm{mM} \mathrm{MgCl}_{2}{ }^{+}$(Invitrogen). For the culture of endothelial cells on the walls of micro-channel, PDMS devices were first sterilised using plasma treatment (Harricks plasma). The channels were then incubated with a $10 \mu \mathrm{g} / \mathrm{ml}$ fibronectin solution for $30 \mathrm{~min}$ at room temperature, rinsed with PBS, and inseminated with a concentrated endothelial cell solution $10^{7}$ cells $/ \mathrm{ml}$.

Cells. Human monocytic THP-1 cells [49] were maintained in Roswell Park Memorial Institute-1640 medium (Invitrogen, Cergy Pontoise, France) supplemented with $20 \mathrm{mM}$ HEPES buffer, $10 \%$ fetal. calf serum (FCS), $2 \mathrm{mM} \mathrm{L}$-glutamine, $50 \mathrm{U} / \mathrm{mL}$ penicillin and $50 \mathrm{mg} / \mathrm{mL}$ streptomycin. Stock cell cultures were passaged twice weekly and maintained at $37^{\circ} \mathrm{C}$ in a humidified atmosphere containing $5 \% \mathrm{CO}_{2}$. For primary $\mathrm{T}$ lymphocytes, total peripheral blood mononuclear cells (PBMCs) were first isolated by ficoll gradient from human blood (Etablissement Français du Sang). PBMCs were stimulated with PHA-L, $1 \mu \mathrm{g} / \mathrm{mL}$ (Roche) for 48-72 hours and subsequently cultured in the RPM1, 10\% FCS, $10 \mathrm{mM}$ Hepes, $2 \mathrm{mM}$ Glutamax in the presence of $50 \mathrm{ng} / \mathrm{mL}$ IL-2 (Miltenyi Biotech). After 6 days of culture, flow cytometry revealed that the cell population was $>95 \%$ $\mathrm{CD}^{3+}$. Cells were used 7-14 days following initial stimulation.

We used Human Umbilical Vein Endothelial Cells (HUVECs) from PromoCell (C-12203) cultivated in Endothelial cell Growth Medium (PromoCell C-22010 and C-39215) with $1 \%$ antibiotic solution $(50 \mathrm{U} / \mathrm{mL}$ penicillin and $50 \mathrm{mg} / \mathrm{mL}$ streptomycin). Culture medium was replaced every 2 days and cells passaged at around $80 \%$ of confluence.

Sera of patients. Patients sera were obtained at the intense care units of Marseilles Hospitals and stored at $-27^{\circ} \mathrm{C}$.

\section{Results and discussion}

Anti-adherent constriction channels. We use here a simple device to test the stiffness of circulating cells and investigate their transit characteristics through narrow capillaries in the absence of adhesion. The device consists of a straight channel, whose width is narrower than the cells' diameter and whose walls have been treated by an anti-adhesive coating. The sequence of pictures in Figure 1(A) presents the entry of a single cell of diameter $12 \mu \mathrm{m}$ in a constriction of rectangular cross section $4 \times 15 \mu \mathrm{m}^{2}$ and length $2250 \mu \mathrm{m}$. The cells are manipulated in the microfluidic device by imposing a constant pressure drop across the circuitry [48]. In a previous study, this device was used to investigate the role of actin organisation and myosin II activity in the different stages of white blood cell trafficking through narrow capillaries [23]. In the entry stage, the entrance time ET, defined as the time interval between the leading edge of the cell crossing the entry into the microchannel and its trailing edge clearing the entry, was found to be strongly dependent on the actin network organisation. In the transit stage, different cell velocities were observed for a given pressure, which proved that our device 
was sensitive enough to measure the minute differences of friction between the cells and the constriction walls that were not detected by previous micropipette experiments [50,51]. We have been able to relate qualitatively the average cell velocity to the thickness of the lubrication film between the cells and the wall of the constriction. This has permitted us to link a slower cell velocity to higher cell stiffness. In the relaxation stage, after cell exit from the constriction, the cells were found to recover their initial spherical shape with a characteristic time of around $50 \mathrm{~s}$ independently of the actin network state or acto-myosin activity level. This surprising result seemed to suggest that the shape relaxation relies on machinery independent of actin. Another explanation might be that changes in the elastic and viscous properties of cell compensate each other yielding roughly similar characteristic of shape relaxation times. On the other hand, the main effect of acto-myosin was found to maintain a sufficient membrane tension and maintain a minimum deformation of cells upon transit. In the absence of acto-myosin activity, we observed in certain conditions a complete unfolding of the reservoir of membrane contained in microvilli. Although further investigations are required to better understand the phenomenon of shape relaxation, these first results have demonstrated how a simple microfluidic constriction permits one to detect detailed characteristics of the cell transit in a capillary at the single cell level. In this paper, we propose to demonstrate the potential of a similar microfluidic single-cell approach to shed new light on a particular pathology, ARDS. Although cell entry, velocity, deformation and relaxation could be studied, we focus here on the entry stage, which is directly linked to the cell stiffness. Indeed, cell stiffness is reported in the literature as a factor that may favour a massive sequestration of leucocytes in the lung microvasculature. We use here model line leucocytes THP-1 and we compare their behaviour before and after their incubation in patients suffering from ARDS. Figure 1(C) presents measurements of single-cell ET as a function of the local pressure drop $\Delta P$ applied to the arrested cells in the constriction of cells before and after 1-hour incubation. ARDS-serum treated cells exhibit significantly longer ET than reference cells, the difference reaching one order of magnitude at $\Delta P=100 \mathrm{~Pa}$. This result proves that leucocytes stiffness may increase significantly in a context of ARDS and that transit times can be greatly increased, which suggests that cell stiffness can play a role in white blood cells sequestration in vivo. Our measurements show an important dispersion of entrance time, but all cells incubated in ARDS patients have stiffness significantly higher than reference cells. Another advantage of our approach using patient sera is that we demonstrate directly that sera contain the biofactors triggering the increase in cell stiffness in ARDS and that the induced effect is relatively fast (less than $1 \mathrm{~h}$ ) and long-lasting (several days for cells continuously cultured in patient sera). In future work, it will be interesting to perform a systematic analysis with a sera library of patients with ARDS to determine a link between sera composition and cell stiffening effect. This should allow us to identify the components responsible for the abnormal mechanical properties of ARDS patients leucocytes and to test new therapies.

Adherent channels covered with endothelial cells. We describe here experiments in channels with dimensions larger than the cell diameter, and with walls covered by endothelial cells, i.e., the cells that coat the lumen of blood vessels. These laminar flow chamber devices are useful for measuring the adhesion properties of cells with an endothelium in the absence of cell deformation and in conditions of hydrodynamic shear 
stress encountered in blood vessels. Such cell adhesion measurements under flow are more physiological than static assays as some adhesive events mediated by selectins and integrins (important class of adhesion molecules) and glycocalyx (polymer regulating the repulsion between cells) occur only in the presence of shear [52,53]. Such measurements under flow also permit a very sensitive detection of weak adhesion events. In practice, white blood cells settle by gravity and are pushed by the flow so that they travel horizontally in the vicinity of the endothelial layer (Figure 2(A)). The frequency and duration of cell arrests under constant hydrodynamic shear stress are monitored. We have compared the behaviour of THP-1 cells on HUVEC endothelial cells monolayer,

- when both are cultured in their respective culture medium (negative reference)

- when HUVEC cells are incubated with an ARDS serum

- when both are incubated with ARDS serum

- when both are incubated with known activators of adhesion, respectively, TNF $\alpha$ [54] and PMA [55] (positive reference).

The adhesion frequency per length travelled by the cell in the vicinity of the endothelium is, respectively, of 2.2, 2.1, 0.9, and 8.7 per millimetre. Detachment curves reported on Figure 2(B) show a similar behaviour of the negative reference and the experiments with sera incubations. The majority of arrest last less than $1 \mathrm{~s}$, whereas for the positive reference the majority of arrests are definitive. Taken together, these results suggest that incubations of THP-1 and endothelial cells with sera of ARDS patients do not induce a significant difference of adhesion when compared with the negative reference. Hence, adhesion may be hardly affected during the early stages of ARDS, contrary to cell stiffness. However, further investigations with sera of different patients, larger statistics and different cell types will be necessary to get a more precise and general response on the role adhesion in cell sequestration during ARDS.

Figure 2 Adherent channels covered with endothelial cells for cell adhesion measurement. (A) Image of THP-1 cells (white contour) settling on a confluent endothelial cell layer under a shear stress of $0.015 \mathrm{dyn} / \mathrm{cm}^{2}$ taken in phase-contrast and in fluorescence. Scale bar corresponds to $50 \mu \mathrm{m}$ and (B) detachment curves for THP-1 and HUVEC incubated in, respectively, culture medium (dots), medium and ARDS-serum (triangle), ARDS-serum and ARDS-serum (squares), and PMA and TNF- $\alpha$ (cross)
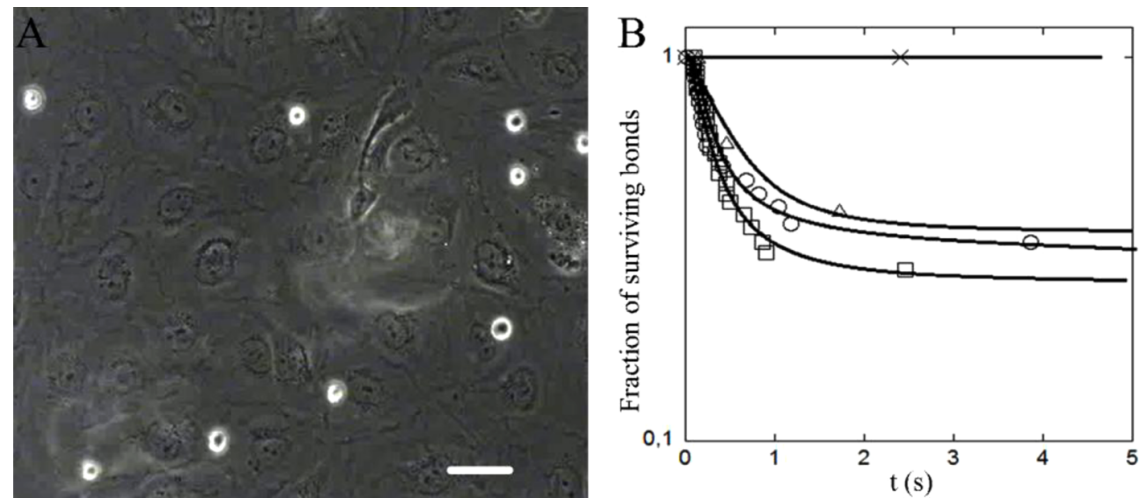
Complex constricted pathway. Experiments in a single and long constriction are not representative of the pathway encountered by a cell transiting in the real blood microvasculature. Indeed, during a single passage in the lung, a cell passes through numerous short constrictions. In the pulmonary microvasculature, the pathway from an arteriole to a venule consists roughly of 50-100 connected constriction segments [50]. As a first approximation of such complex microvasculature pathway, we have designed a microfluidic circuitry made of a succession of 50 crenelled constrictions of width $4 \mu \mathrm{m}$ and length $20 \mu \mathrm{m}$ connected by segments of width $20 \mu \mathrm{m}$ and length $20 \mu \mathrm{m}$. Such a device was used in a previous work to study the passage of single white blood cells through interconnected capillary segments. We had shown that the time needed to enter the first constriction was much larger than the entry times in subsequent constrictions [23]. This was explained by the characteristic time of cell shape relaxation (around $50 \mathrm{~s}$ ), which is much longer than the travel time between the exit of a constriction and the arrival at the next constriction entry $(<1 \mathrm{~s})$ in physiological flow rate conditions. Hence, for all constrictions but the first one, the cell is already deformed before entry and this pre-deformed shape shortens its entry time in subsequent constrictions. These findings suggested that the transit time was not significantly different in a single constriction channel (Figure 1) and in a multiple constrictions channel of same global length (Figure 3(A)). In this view, the single constriction device was relevant to estimate the transit times in complex microvasculature and can be used to analyse cell transit in pathological conditions like in ARDS. However, further experiments have demonstrated that more complex transit behaviours are also possible in multiple constriction pathways [48]. When the cell orientation is perturbed between two constrictions, the benefit of shape pre-deformation gets lost. The cell entry in the next constriction requires a time-consuming shape deformation, and the entry time is then comparable with the cell entry time in the first capillary. In this context, it appears that a transit time in multiple constrictions can be much longer than the transit time in a single constriction. Roughly, the total transit time is multiplied by the number of occurrence of cell orientation perturbation between two constrictions.

The probability of cell orientation perturbations in symmetric crenels (Figure 3(A) is intrinsically low and perfect symmetry is obviously not relevant to describe real microvasculature. Therefore, to challenge further the idea that cell orientation perturbations may induce long retention times, we have designed crenelled channels with broken symmetry to force a random cell orientation in between two constrictions (Figure 3(B)-(D)). In this new device, the outlets of the constrictions have one side making an angle of $90^{\circ}$ and the other side an angle of $45^{\circ}$. In these asymmetric crenels, we have found that the transit of a cell differs strongly depending on its stiffness, the stiffness of each individual cell being assessed by its entry time in the first constriction (as illustrated in Figure 3(B)-(D)). Experiments with cells of different stiffness can be made with cells without specific treatment because the distribution of stiffness in a cell population is wide [56] enough to allow an exploration of stiffness effects. For the most compliant cells, we observed like in symmetric crenels that the ET is much longer in the first constriction than in the subsequent ones. However, at each constriction exit, the cell trajectory is deviated upwards from the median pathway and the cell orientation is also shifted, the cell head pointing upward. The cells are deformed and tilted after exiting each constriction and they hit the upper part of the next constriction entry before entering it. 
This shows that the broken symmetry of crenels fulfils the objective to perturb the favourable and straight passage of pre-deformed cells. However, compliant cells do not block at constriction entrances but still enter very fast. Indeed, just before constriction entry, the orientation of a cell changes rapidly to present the smallest section to the constriction entry, which corresponds to a favourable orientation for fast entry. This effect implies a rapid cell rotation either opposite to the cell rotation at the constriction exit and the cell enters then head-first in the constriction (Figure 3(B)), or a complete $180^{\circ}$ rotation and the cell enters then tail-first in the constriction (Figure 3(C)). In both cases, the cell transit is perturbed by the asymmetry of channels but the global transit times are hardly affected when compared with transit in symmetric crenels. The situation is completely different with rigid cells. In this case, the trajectory and orientation is also strongly perturbed at the exit of each constriction and the entry in the next constriction can be problematic. When cells present their largest section at the approach of a constriction entry, they are temporarily stopped. A strong and time-consuming deformation of the cell is necessary before the cell can travel through the constriction. Hence, for rigid cells, the asymmetry of multiple constrictions channels leads to a great increase in the global transit time.

Figure 3 Multiple constriction channel to mimic complex microvasculature. Effect of symmetry. Image sequences of white blood cells THP-1 during transit through two successive crenelled constrictions with symmetric outelts (A) and asymmetric outlets (B-D). Sequences (B, C) correspond to compliant cells and (D) to stiff cells. The red dot materialises a physical point on a cell membrane to help visualisation of cell orientation. Cells enter fast ( $<20 \mathrm{~ms})$ in the following constriction either head-first (B), or tail-first after a $180^{\circ}$ rotation $(\mathrm{C})$, whereas they stop for a long time ( $\left.>1000 \mathrm{~ms}\right)$ after a $90^{\circ}$ rotation in (D). Local pressures are $\Delta P=100 \mathrm{~Pa}$ in $(\mathrm{A}-\mathrm{C})$ and $150 \mathrm{~Pa}$ in (D). Scale bar corresponds to $10 \mu \mathrm{m}$ (see online version for colours)
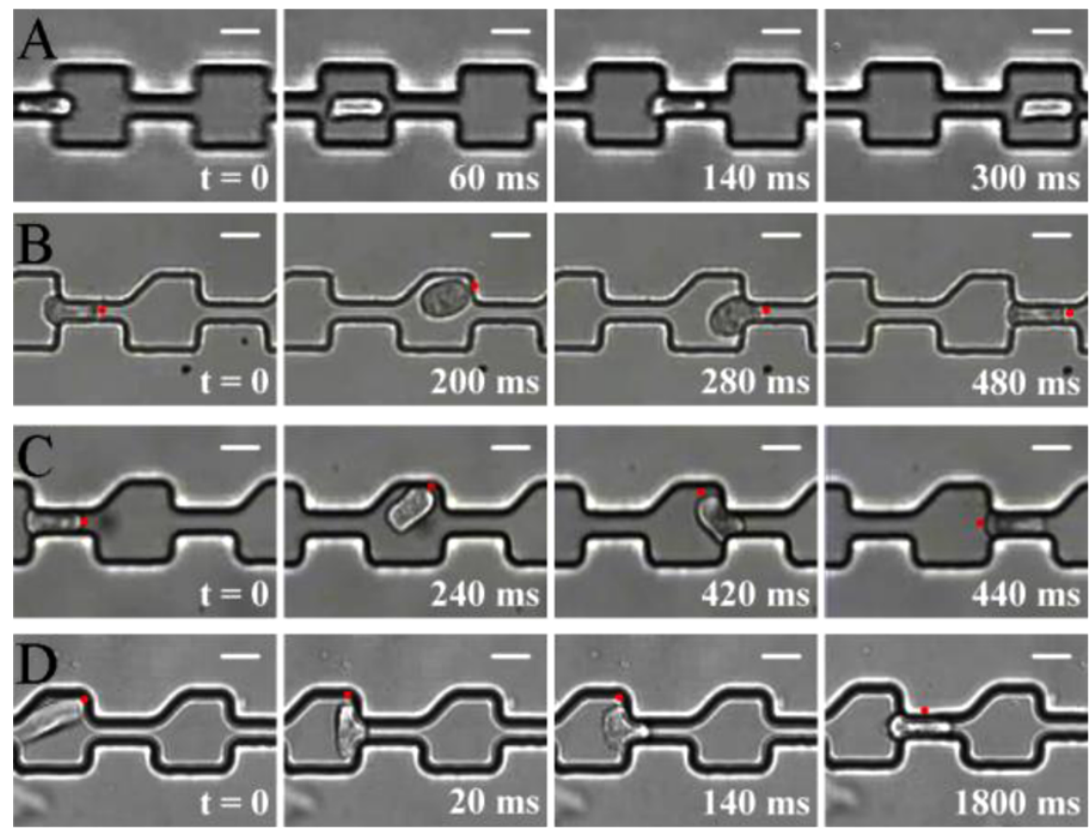
We have then compared the behaviour of cells incubated in sera of ARDS patient in symmetric and asymmetric crenels. With similar driving pressures, cells travel fast along a median pathway in symmetric channels whereas arrests are observed in asymmetric crenels, which corresponds to the behaviour observed with rigid cells. This result suggests that asymmetry in multiple constrictions in series can favour longer cell transit times and sequestration with ARDS patients as observed in vivo. However, further investigations are necessary to determine whether asymmetry plays a significant role in vivo. Indeed, the entries of blood capillaries do not have right angle shapes like our crenelled channels. It is known that the smooth capillary entrance, i.e., larger radii of curvatures facilitates the entrance of cells in constriction [50]. Moreover, a smooth entrance is also expected to guide the movement of pre-deformed cells towards a favourable orientation in the approach of a constriction. Smooth entry effects favouring fast entry can counter-balance potential asymmetry effects favouring longer transit times. To probe the result of these competing effects in vivo, one should design new devices with channels mimicking more precisely the physiologic asymmetry and curvature of microvasculature.

Porous channels. It is accepted that the massive blockage of leucocyte in the lung microvasculature plays a role in triggering the deterioration of pulmonary function. However, the severe damage to the alveolar lumen tissue is the result of a late inflammatory activity by sequestered white blood cells. The complex inflammatory processes involving leucocytes interactions with the endothelium, deterioration of endothelium and transmigration of leucocytes from the blood system into tissues and alveoli lumen have not been observed in vivo in the context of ARDS. An in vitro approach with microfluidics opens new opportunities to investigate such events. The challenge consists here in fabricating micro-vessels covered by endothelial cells and displaying porous walls to allow leucocyte-endothelium interactions and also transmigration of white blood cells through the vessel walls. We present a prototype of such a microfluidic model (Figure $4(\mathrm{~A})$ and (B)). Wall porosity is mimicked with narrow side channels (width $2 \mu \mathrm{m}$ ) perpendicular to the central channel mimicking the blood micro-vessel. In a typical experiment, fluid and cells are injected in the central channel only. The collector channels collect only the fraction of fluid and cells that have crossed the wall porosity. We present on Figure 4(B) a first application of this prototype. The walls of the device are covered with ICAM adhesion molecules to obtain a model surface for adhesion and migration of leucocytes via integrin-dependent motility. With this device, one can study in a single experiment the adhesion kinetics of leucocytes, their migration properties on a $2 \mathrm{D}$ surface under physiological flow conditions, and the transmigration of cells through the walls, which involves 3D motility mechanisms (Figure 4(C)). Such experiments will permit us to investigate the role of hydrodynamic shear stress on 2D motility and the cellular processes involved in the transition between 2D and 3D motility modes. In another experiment, the walls of the central channel have been inseminated with HUVEC endothelial cells. After several days of culture, the channels are covered with a confluent layer of endothelial cells (Figure 4(B)). This device will be useful in future work to investigate further the physiopathology of the lung microvasculature in the context of different inflammatory diseases such as ARDS, sepsis or pneumonia. It will also be useful to investigate infections of the microvasculature by pathogens. For instance, we are interested in the 
infection of human brain microvasculature by bacteria Neisseiria meningitidis during severe meningitis. Current in vitro models include infecting cells in a Petri dish for in vitro assays and injecting massive doses of infectious agents in mice for in vivo models. Although these strategies have yielded important information, they fail to reproduce important conditions found during the infection process and therefore only give a partial view. The in vitro microfluidic model will allow observation of all the stages of the infection, from the bacteria arrest, colony growth, permeabilisation of the endothelial barrier and transmigration of bacteria through the channel walls in the collector channels.

Figure 4 Porous microvessel. (A) Schematic of the device showing the central channel mimicking a blood vessel, the multiple side channels mimicking wall porosity (cross section of $3 \times 3 \mu \mathrm{m}^{2}$ ), and the two collector channels on the side. (B) Micrograph of a porous microvessel device with endothelial cells. (C) Porous microvessel with walls covered by ICAM molecules, allowing observation of the migration of leucocytes (T-cells) on a planar surface in 2D (upper cell) as well as the transmigration in the confined 3D environment of the porosity channels (lower cell). Scale bars correspond to $20 \mu \mathrm{m}$ (see online version for colours)
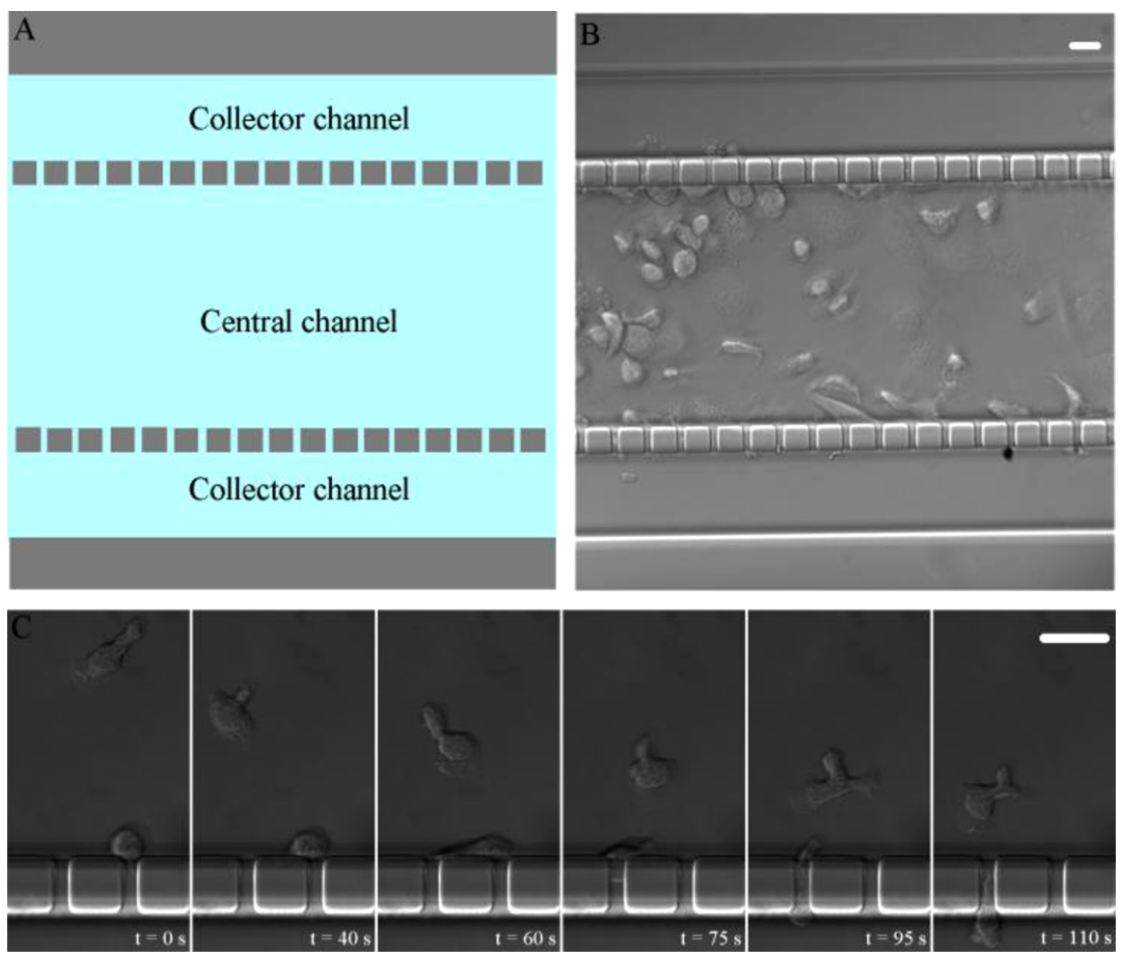

\section{Conclusion and perspectives}

We have presented an innovative approach to studying pathologies in the blood microcirculation based on a series of microfluidic tools. These tools permit to observe 
circulating cells (immune cells, circulating tumour cells and pathogenic bacteria) in biomimetic vessels and characterise their rheological behaviour, their properties of passage in a constriction under flow, their interactions with the endothelium and their transmigration across the endothelial barrier. Such analysis is useful for a wide range of pathologies like sepsis, leukaemia, pneumonia, metastasis, respiratory distress syndrome or meningitis. Beyond academic investigations, the microfluidic in vitro approach is also advantageous for medical applications for the possibility to screen drugs without constraints. Finally, the coupling of microfluidic devices to microscopy modes such as Wet-SEEC [57,58], RICM [59-62], TIRF [5] or ultraresolution PALM microscopy [6] permits to envision the study of biological mechanisms in biomimetic microvasculatures at both the nanometric and molecular scale.

\section{Acknowledgements}

The authors thank Cyprien Gay for helpful discussions and acknowledge the Rhodia Company (LOF, Pessac, France) and CINAM (CNRS UPR 3118, Marseille, France) for technical support with microfabrication. Postdoctoral fellowship of TL was supported by ANR 'Bugs in Flow' and Doctoral fellowships of PP by both the Region PACA and the company CAPSUM-SAS. AL, MPV and OT acknowledge support from the Association pour la Recherche sur le Cancer (ARC).

\section{References}

1 Zernike, F. (1942) 'Phase contrast, a new method for the microscopic observation of transparent objects', Physica, Vol. 9, No.10, pp.974-986.

2 Nomarski, G. (1960) Interferential Polarizing Device for Study of Phase Objects, US Patent Office, 2, 924, 142.

3 Lessor, D.L., Hartman, J.S. and Gordon, R.L. (1979) 'Quantitative surface topography determination by Nomarski reflection microscopy, I. Theory', J. Opt. Soc. Am., Vol. 69, pp.357-366.

4 Helmchen, F. and Denk, W. (2005) 'Deep tissue two-photon microscopy', Nat. Methods, Vol. 2, pp.932-940.

5 Axelrod, D. (1981) 'Cell surface contacts illuminated by total internal reflection fluorescence', J. Cell Biol., Vol. 89, pp.141-145.

6 Betzig, E., Patterson, G.H., Sougrat, R., Lindwasser, O.W., Olenych, S., Bonifacino, J.S., Davidson, M.W., Lippincott-Schwartz, J. and Hess, H.F. (2006) 'Imaging intracellular fluorescent proteins at nanometer resolution', Science, Vol. 313, pp.1642-1645.

7 Looney, M.R., Thornton, E.E., Sen, D., Lamm, W.J., Glenny R.W. and Krummel, M. (2011) 'Stabilized imaging of immune surveillance in the mouse lung', Nat. Methods, Vol. 8, No.1, pp.91-96.

8 El-Ali, J., Sorger, P.K. and Jensen, K.F. (2006) 'Cells on chips', Nature, Vol. 442, pp.403-411.

9 Salieb-Beugelaar, G.B., Simone, G., Arora, A., Philippi, A. and Manz, A. (2010) 'Latest developments in microfluidic cell biology and analysis systems', Anal. Chem., Vol. 82, No. 12 , pp.4848-4864. 
10 Velve-Casquillas, G., Le Berre, M., Piel, M. and Tran, P.T. (2010) 'Microfluidics tools for cell biological research', Nanotoday, Vol. 5, pp.28-47.

11 Lipowsky, H.H., Kovalcheck, S. and Zweifach, B.W. (1978) 'The distribution of blood rheological parameters in the microvasculature of cat mesentery', Circ. Res., Vol. 43, No. 5, pp.738-749.

12 Yoshida, K., Ryoichi, K., Qin, W. and Doerschuk, C.M. (2006) 'Neutrophil Cytoskeletal rearrangements during Capillary Sequestration in Bacterial Pneumonia in Rats', Am. J. Respir. Crit. Care. Med., Vol. 174, pp.689-698.

13 Hotchkiss, S.R. and Karl, I.E. (2003) 'The pathophysiology and treatment of sepsis', N. Engl. J. Med., Vol. 348, No. 2, pp.138-150.

14 Hogg, J.C. (1987) 'Neutrophil kinetics and lung injury', Physiological Review, Vol. 67, No. 4, pp.1249-1295.

15 Wong, C.W., Song, C., Grimes, M.M., Fu, W.L., Dewhirst, M.W., Muschel, R.J. and Al-Mehdi, A.B. (2002) 'Intravascular location of breast cancer cells after spontaneous metastasis to the lung', Am. J. Pathol., Vol. 161, No. 3, pp.749-751.

16 Lam, W.A., Rosenbluth, M.J. and Fletcher, D.A. (2008) 'Increased leukaemia cell stiffness is associated with symptoms of leucostasis in paediatric acute lymphoblastic leukaemia', Br. J. Haematology, Vol. 142, No. 3, pp.497-501.

17 Alon, R., Aker, M., Feigelson, S., Sokolovsky-Eisenberg, M., Staunton, D.E., Cinamon, G., Grabovsky, V., Shamri, R. and Etzioni, A. (2003) 'A novel genetic leukocyte adhesion deficiency in subsecond triggering of integrin avidity by endothelial chemokines results in impaired leukocyte arrest on vascular endothelium under shear flow', Blood, Vol. 101, No. 11, pp.4437-4445.

18 Robert, P., Canault, M., Farnarier, C., Nurden, A., Grosdidier, C., Barlogis, V., Bongrand, P., Pierres, A., Chambost, H. and Alessi, M.C. (2011) 'A novel Leukocyte adhesion deficiency III variant: kindlin-3 deficiency results in integrin and non-integrin-related defects in different steps of leukocyte adhesion', J. Immunol., in press.

19 Mairey, E., Genovesio, A., Donnadieu, E., Bernard, C., Jaubert, F., Pinard, E., Seylaz, J., Olivo-Marin, J.C., Nassif, X. and Dumenil, G. (2006) 'Cerebral microcirculation shear stress levels determine Neisseria meningitides attachment sites along the blood-brain barrier', J. Exp. Med., Vol. 203, No. 8, pp.1939-1950.

20 Gross, S. and Walden, P. (2008) 'Immunosuppressive mechanisms in human tumors: Why we still cannot cure cancer', Immunol. Lett., Vol. 116, pp.7-14.

21 Van der Meer, A.D., Poot, A.A., Duits, M.H.G., Feijen, J. and Vermes, I. (2009) 'Microfluidic technology in vascular research', J. Biomed. Biotechnol., Vol. 2009, Article ID 823148, pp.1-10.

22 Nishino, N., Tanaka, H., Ogura, H., Inoue, Y., Koh, T., Kieko Fujita, B.S. and Hisashi Sugimoto, M.D. (2005) 'Serial changes in white blood cells deformability and whole blood rheology in patients with sepsis or trauma', J. Trauma, Vol. 59, No. 6, pp.1425-1431.

23 Gabriele, S., Benoliel, A.M., Bongrand, P. and Theodoly, O. (2009) 'Microfluidic investigation reveals distinct roles for actin cytoskeleton and Myosin II activity in capillary white blood cell trafficking', Biophys. J., Vol. 96, pp.4308-4318.

24 Rosenbluth, M.J., Lam, W.A. and Fletcher, D.A. (2008) 'Analyzing cell mechanics in hematologic diseases with microfluidic biophysical flow cytometry’, Lab Chip, Vol. 8, No. 7 , pp.1062-1070.

25 Chaw, K.C., Manimaran, M., Francis, E.H. and Swaminathan S. (2006) 'A quantitative observation and imaging of single tumor cell migration and deformation using a multi-gap microfluidic device representing the blood vessel', Microvascular Research, Vol. 72, pp.153-160. 
26 Yap, B. and Kamm, R.D. (2005) 'Mechanical deformation of neutrophils into narrow channels induces pseudopod projection and changes in biochemical properties', J. Appl. Physiol., Vol. 98, pp.1930-1939.

27 Shelby, J.P., White, J., Ganesan, K., Rathod, P.K. and Chiu, D.T. (2003) 'A microfluidic model for single-cell capillary obstruction by plasmodium falciparum-infected erythrocytes', PNAS, Vol. 100, No.25, pp.14618-14622.

28 Hawkins, R.J., Piel, M., Faure-Andre, G., Lennon-Dumenil, A.M., Joanny, J.F., Prost, J. and Voituriez, R. (2009) 'Pushing off the walls: a mechanism of cell motility in confinement', Phys. Rev. Lett., Vol. 102, No. 058103, pp.1-5.

29 Jacobelli, J., Friedman, R.S., Conti, M.A., Lennon-Dumenil, A.M., Piel, M., Sorensen, C.M., Adelstein, R.S. and Krummel, M.F. (2010) 'Confinement-optimized three-dimensional T cell amoeboid motility is modulated via myosin IIA-regulated adhesions', Nat. Immunol., Vol. 11, No. 10, pp.953-961.

30 Chaw, K.C., Manimaran, M., Tay, F.E.H. and Swaminathan, S. (2006) 'Three-dimensional (3D) extra-cellular matrix coating of a microfluidic device', J. Phys.: Conference Series, Vol. 34, pp.747-751.

31 Van der Meer, A.D., Poot, A.A., Duits, M.H.G., Feijen, J. and Vermes. I. (2009) 'Microfluidic Technology in vascular research', J. Biomed. Biotechnol., Vol. 2009, ID.823148, pp.1-10.

32 Young, E.W.K. and Simmons, C.A. (2010) 'Macro- and microscale fluid flow systems for endothelial cell biology', Lab Chip, Vol. 2010, No. 10, pp.143-160.

33 Song, J.W., Gu, W., Futai, N., Warner, K.A., Nor, J.E. and Takayama, S. (2005) 'Computer-controlled microcirculatory support system for endothelial cell culture and shearing', Anal. Chem., Vol. 77, No.13, pp.3993-3399.

34 Tkachenko, E., Gutierrez, E., Ginsberg, M.H. and Groisman, A. (2009) 'An easy to assemble microfluidic perfusion device with a magnetic clamp', Lab Chip, Vol. 9, No. 8, pp.1085-1095.

35 Khan, O.F. and Sefton, M.V. (2011) 'Endothelial cell behaviour within a microfluidic mimic of the flow channels of a modular tissue engineered construct', Biomed. Microdevices, Vol. 13, No.1, pp.69-87.

36 Van der Meer, A.D., Poot, A.A., Feijen, J. and Vermes, I. (2010) 'Analyzing shear stress-induced alignment of actin filaments in endothelial cells with a microfluidic assay', Biomicrofluidics, Vol. 4, No. 1, 011103, pp.1-10.

37 Song, J.W., Cavnar, S.P., Walker, A.C., Luker, K.E., Gupta, M., Tung, Y.C., Luker, G.D. and Takayama, S. (2009) 'Microfluidic endothelium for studying the intravascular adhesion of metastatic breast cancer cells', PLOS One, Vol. 4, No. 6, p.e.5756.

38 Shao, J.B., Wu, L., Wu, J.Z., Zheng,Y.H., Zhao, H., Jin, Q.H. and Zhao, J.L. (2009) 'Integrated microfluidic chip for endothelial cells culture and analysis exposed to a pulsatile and oscillatory shear stress', Lab Chip, Vol. 9, No.21, pp.3118-3125.

39 Seok, C., Ryo, S., Peter, J.M., Chen-Rei, W., Vernella, V. and Roger, D. (2009) 'Cell migration into scaffolds under co-culture conditions in a microfluidic platform', Lab Chip, Vol. 2009, No. 9, pp.269-275.

40 Huh, D., Matthews, B.D., Mammoto, A., Montoya-Zavala, M., Yuan Hsin, H. and Ingber, D.E. (2010) 'Reconstituting organ-level lung functions on a chip', Science, Vol. 328, No. 5986, pp.1662-1668.

41 Ashbaugh, D.G., Bigelow, D.B., Petty, T.L. and Levine, B.E. (1967) 'Acute respiratory distress in adults', Lancet, Vol. 2, pp.319-323.

42 Ware, L.B. and Matthay, M.A. (2000) 'The acute respiratory distress syndrome', N. Engl. J. Med., Vol. 342, No. 18, pp.1334-1349. 
43 Skoutelis, A.T., Vassilios, K., George, M.A., Kyriakos, I.K., Yannis, F.M. and Harry, P.B. (2000) 'Neutrophil deformability in patients with sepsis, septic shock, and adult respiratory distress syndrome', Crit. Care. Med., Vol. 2000, No. 28, pp.2355-2359.

44 Rosenbluth, M.J., Lam, W.A. and Fletcher, D.A. (2008) 'Analyzing cell mechanics in hematologic diseases with microfluidic biophysical flow cytometry', Lab Chip, Vol. 8, No. 7, pp.1062-1070.

45 Ibbotson, G.C., Doit, C., Kaur, J., Varinder, G., Lena, O., Todd, F. and Kubes, P. (2001) 'Functional $\alpha 4$-integrin: A newly identified pathway of neutrophil recruitment in critically ill septic patients', Nat. Med., Vol. 7, No. 4, pp.465-470.

46 Sion, M.L., David, F.T., Lesley, B., Susan, D.B., David, J.C., Pearson, D.J. and Kenneth, A.B. (2009) 'Plasma from patients with sepsis up-regulates the expression of CD49d and CD64 on blood neutrophils', Am. J. Respir. Cell Mol. Biol., Vol. 40, pp.724-732.

47 Xia, Y. and Whitesides, G.M. (1998) 'Soft lithography', Angew. Chem. Int. Ed., Vol. 37, pp.550-575.

48 Gabriele, S., Versaevel, M., Preira, P. and Theodoly, O. (2010) 'A simple microfluidic method to select, isolate, and manipulate single-cells in mechanical and biochemical assays', Lab Chip, Vol. 10, pp.1459-1467.

49 Tsuchiya, S., Yamabe, M., Yamaguchi, Y., Kobayashi, Y. and Konno, T. (1980) 'Establishment and characterization of a human acute monocytic leukemia cell line (THP-1)', Int. J. Cancer, Vol. 26, pp.171-176.

50 Bathe, M., Shirai, A., Doerschuk, C.M. and Kamm, R.D. (2002) 'Neutrophil transit times through pulmonary capillaries: the effects of capillary geometry and fMLP-stimulation', Biophys. J., Vol. 83, pp.1917-1933.

51 Needham, D. and Hochmuth, R.M. (1990) 'Rapid flow of passive neutrophils into a 4 microns pipette and measurement of cytoplasmic viscosity', J. Biomech. Eng., Vol. 112, No. 3, pp.269-276.

52 Finger, E.B., Puri, K.D., Alon, R., Lawrence, M.B., VonAndrian, U.H. and Springer, T.A. (1996) 'Adhesion through L-selectin requires a threshold hydrodynamic shear', Nature, Vol. 379, No. 6562, pp.266-269.

53 Sabri, S., Soler, M., Foa, C., Pierres, A., Benoliel, A. and Bongrand, P. (2000) 'Glycocalyx modulation is a physiological means of regulating cell adhesion', J. Cell Sci., Vol. 113, pp.1589-1600.

54 Harlan, J.M. and Liu. D.Y. (1992) Adhesion - Its Role in Inflammatory Diseases, W.H. Freeman \& Co, New York, pp.117-150

55 Kucik, D.F., Dustin, M.L., Miller, J.M. and Brown, E.J. (1996) 'Adhesion-activating phorbol ester increases the mobility of leukocyte integrin LFA-1 in cultured lymphocytes', J. Clin. Invest., Vol. 97, pp.2139-2144.

56 Balland, M., Desprat, N., Icard, D., Féréol, S., Asnacios, A., Browaeys, J., Hénon, S. and Gallet, F. (2006) 'Power laws in microrheology experiments on living cells: comparative analysis and modeling', Phys. Rev., Vol. 74, No. .2, No. 021911.

57 Ausserre, D. and Valignat, M.P. (2006) 'Wide-field optical imaging of surface nanostructures', Nano Lett., Vol. 6, pp.1384-1388.

58 Valignat, M.P., Ducret, A., Métivier, M., Médard, N., Gabriele, S. Mignot, T. and Theodoly O. The Underwater Nanoworld on a Microscope Stage, in preparation.

59 Curtis, A.S. (1964) 'The mechanism of adhesion of cells to glass. A study by interference reflection Microscopy', J. Cell Biol., Vol. 20, pp.199-215.

60 Gingell, D. and Todd, I. (1979) 'Interference reflection microscopy. A quantitative theory for image interpretation and its application to cell-substratum separation measurement', Biophys. J., Vol. 26, pp.507-526. 
Microfluidic tools to investigate pathologies in the blood microcirculation 547

61 Rädler, J. and Sackmann, E.J. (1993) 'Imaging optical thicknesses and separation distances of phospholipid vesicles at solid surfaces', J. Phys. II, Vol. 3, pp.727-748.

62 Theodoly, O., Huang, Z.H. and Valignat, M.P. (2010) 'New modeling of reflection interference contrast microscopy including polarization and numerical aperture effects: application to nanometric distance measurements and object profile reconstruction', Langmuir, Vol. 26, No. 3, pp.1940-1948. 